\title{
O problema do mapeamento no plano
}

Josimar da Silva Rocha

\author{
Rogério César dos Santos
}

\author{
Hudson Rodrigues Armando
}

\section{Resumo}

O objetivo deste artigo é mostrar uma forma de se fazer mapeamento do plano, no sentido de que, se conhecemos as distâncias duas a duas entre quatro pontos do plano, é possível determinarmos as coordenadas de tais pontos. O estudo do problema aqui apresentado pode servir de atividade lúdica que aplica e contextualiza resultados básicos da Geometria Euclidiana e da Geometria Analítica.

Palavras-chave: Mapeamento; Distância; Teorema de Pitágoras.

\begin{abstract}
The aim of this paper is to show a way to map the plane, in the sense that if we know the distances two by two between four points of the plane, we can determinate the coordinates of such points. The study of the problem presented here can serve as a playful activity that applies and contextualizes basic results of Euclidean Geometry and Analytical Geometry.
\end{abstract}

Keywords: Mapping; Distance; Pythagorean theorem.

\section{Introdução}

O artigo apresentado traz um interessante problema que mostra uma aplicação prática do estudo das coordenadas no plano cartesiano. O emprego das coordenadas no plano tem uma dupla finalidade, como apontada em [2]: a primeira é atribuir um significado geométrico (e com isso dar um maior conteúdo intuitivo) a fatos de natureza numérica. A segunda finalidade vai no sentido oposto ao da primeira: recorre-se a coordenadas a fim de resolver problemas de Geometria.

As ferramentas teóricas utilizadas não vão além das que são empregadas por professores do ensino médio ou técnico nas disciplinas de Geometria Plana e Analítica. Assim, o estudo do problema aqui proposto pode ser utilizado em salas de aula como um exemplo de aplicação dessas disciplinas.

\section{O Problema}

Para permitir uma visualização lúdica do problema, vamos considerar que os pontos que formam um plano sejam cidades numeradas de 1 até $n$, e seja $M=\left(d_{i j}\right)$ a matriz que representa a distância entre as cidades, ou seja, para cada $i, j \in\{1,2, \cdots, n\}$, o elemento de matriz $d_{i j}$ corresponderá à 
distância euclidiana entre as cidades $i$ e $j$, de tal forma que suas distâncias satisfazem a desigualdade triangular estrita:

$$
\left\{\begin{array}{c}
d_{i j}<d_{j k}+d_{i k} \\
d_{j k}<d_{i j}+d_{i k} \\
d_{i k}<d_{i j}+d_{j k}
\end{array}\right.
$$

Sem perda de generalidade, podemos fixar a cidade $i$ na origem do plano cartesiano, ou seja, no ponto $P_{i}=(0,0)$. A cidade $j$ será fixada em $P_{j}=\left(0, d_{i j}\right)$ e a cidade $k$ em $P_{k}=\left(x_{k}, y_{k}\right)$. Representaremos a distância entre as cidades $i$ e $k$ como $d_{i k}=d\left(P_{i}, P_{k}\right)$ e, de forma análoga, a distância entre as cidades $j$ e $k$ será escrita como $d_{j k}=d\left(P_{j}, P_{k}\right)$. Note que, para calcular a distância entre dois pontos num plano, não faremos mais do que simplesmente aplicar um dos mais importantes e úteis teoremas da Geometria Euclidiana Plana: O "Teorema de Pitágoras"[1]. Dessa forma, mostraremos que é possível obter a localização (ou seja, as coordenadas) de uma cidade $t$ qualquer no plano cartesiano de forma biunívoca a partir das distâncias tomadas duas a duas entre os pontos $P_{i}, P_{j}, P_{k}$ e $P_{t}$, como ilustrado na Figura 1

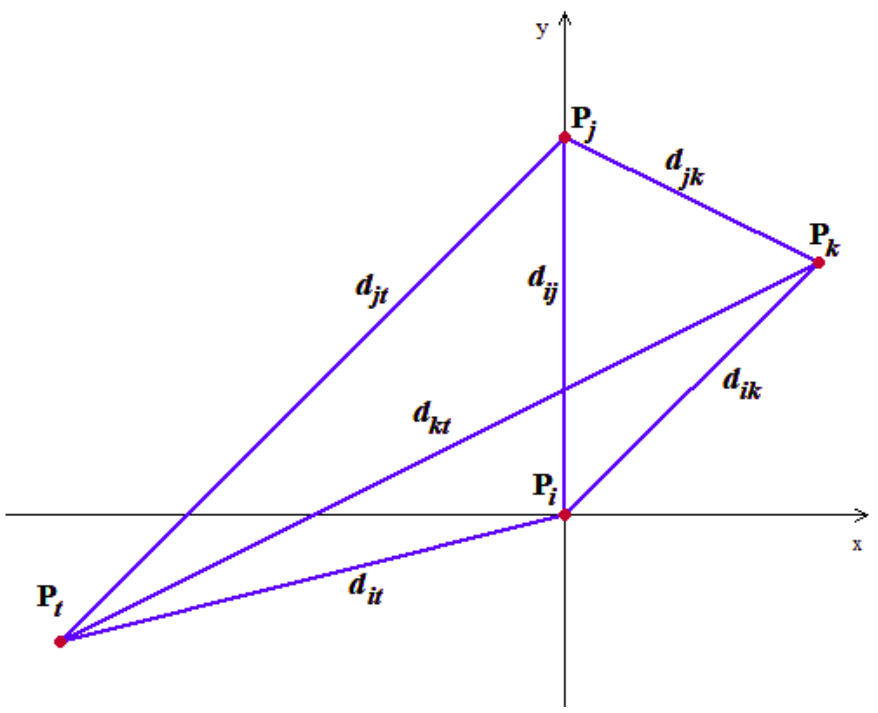

Figura 1: Cidades $P_{i}, P_{j}, P_{k}$ e $P_{t}$ 


\subsection{Um Resultado Intermediário}

Como vimos, o objetivo deste artigo é provar que as coordenadas de uma cidade ficam determinadas dadas as distâncias duas a duas entre ela e mais três cidades distintas. Se, porém, a cidade da qual queremos descobrir as coordenadas possui abscissa positiva, bastam apenas mais duas cidades distintas. É o que provaremos nesta seção. Este caso particular será importante para a prova do caso geral que veremos na seção 3 Iremos provar agora que uma cidade $P_{k}$ que tenha abscissa $x_{k}>0$ fica unicamente determinada quando são dadas apenas as distâncias $d_{i j}, d_{j k}$ e $d_{i k}$. Vejamos:

Observando a Figura (1), vemos as posições dos pontos $P_{i}=(0,0), P_{j}=\left(0, d_{i j}\right)$ e $P_{k}=\left(x_{k}, y_{k}\right)$. Assim, temos:

$$
d_{i k}^{2}=d\left(P_{i}, P_{k}\right)^{2}=x_{k}^{2}+y_{k}^{2}
$$

e

$$
d_{j k}^{2}=x_{k}^{2}+\left(d_{i j}-y_{k}\right)^{2}
$$

Desse modo,

$$
d_{j k}^{2}=d\left(P_{j}, P_{k}\right)^{2}=x_{k}^{2}+y_{k}^{2}-2 y_{k} d_{i j}+d_{i j}^{2} .
$$

Subtraindo a equação (2) de (3), obtemos

$$
2 y_{k} d_{i j}-d_{i j}^{2}=d_{i k}^{2}-d_{j k}^{2} .
$$

O termo $d_{i j}$ deve ser tal que $d_{i j}>0$ (pois as cidades $i$ e $j$ são distintas). Dessa forma, podemos isolar a variável $y_{k}$ da equação anterior e obter:

$$
y_{k}=\frac{d_{i j}^{2}+d_{i k}^{2}-d_{j k}^{2}}{2 d_{i j}}
$$

Assim, obtemos a coordenada $y_{k}$ em função das distâncias $d_{i j}, d_{j k}$ e $d_{i k}$. Para fazer o mesmo em relação à coordenada $x_{k}$, podemos recorrer à equação (2), dada por:

$$
d_{i k}^{2}=x_{k}^{2}+y_{k}^{2}
$$

onde podemos isolar a coordenada $x_{k}$. Como ela é positiva por hipótese, podemos isolá-la assim:

$$
x_{k}=\sqrt{d_{i k}^{2}-y_{k}^{2}}
$$

Observe que poderíamos ter chegado aos mesmos resultados se o ponto $P_{j}$ tivesse ordenada negativa. Isso mostra a possibilidade de se determinar univocamente $P_{k}=\left(x_{k}, y_{k}\right)$ dadas as distâncias duas a duas entre as cidades $i, j$ e $k$, porém com a restrição $x_{k}>0$, isto é, com a abscissa de $P_{k}$ positiva. 


\section{A Prova do Resultado Principal}

Agora sim vamos determinar as coordenadas de uma quarta cidade $P_{t}$ qualquer, sem restrição de suas coordenadas, dadas as distâncias duas a duas entre $i, j, k$ e $t$, onde estamos nas mesmas hipóteses do enunciado do problema inicial como mostra a Figura (1):

- $P_{k}=\left(x_{k}, y_{k}\right)$, onde $x_{k}>0$

- $P_{i}=(0,0)$ e $P_{j}=\left(0, d_{i j}\right)$

Seja $t$ uma cidade cujas coordenadas no plano cartesiano compõem o ponto $P_{t}=\left(x_{t}, y_{t}\right)$. Considerando a distância da cidade $t$ com relação às cidades $i, j$ e $k$, obtemos:

$$
\begin{gathered}
d\left(P_{t}, P_{i}\right)^{2}=d_{i t}^{2}=x_{t}^{2}+y_{t}^{2}, \\
d\left(P_{t}, P_{j}\right)^{2}=\left(-x_{t}\right)^{2}+\left(-y_{t}+d_{i j}\right)^{2}, \\
d\left(P_{t}, P_{j}\right)^{2}=d_{t j}^{2}=x_{t}^{2}+y_{t}^{2}-2 y_{t} d_{i j}+d_{i j}^{2} .
\end{gathered}
$$

Também temos

$$
d\left(P_{t}, P_{k}\right)^{2}=\left(x_{k}-x_{t}\right)^{2}+\left(y_{k}-y_{t}\right)^{2} .
$$

Assim,

$$
d\left(P_{t}, P_{k}\right)^{2}=d_{t k}^{2}=x_{t}^{2}-2 x_{t} x_{k}+x_{k}^{2}+y_{t}^{2}-2 y_{t} y_{k}+y_{k}^{2} .
$$

Por $(7)$ e (8),

$$
y_{t}=\frac{d_{i j}^{2}+d_{i t}^{2}-d_{t j}^{2}}{2 d_{i j}} .
$$

Ou seja, já temos a ordenada $y$ de $P_{t}$. Para obtermos a abscissa de $P_{t}$, podemos rearranjar os termos da equação (9) para observar que:

$$
d_{t k}^{2}=\underbrace{x_{t}^{2}+y_{t}^{2}}_{d_{i t}^{2}}-2 x_{t} x_{k}-2 y_{t} y_{k}+\underbrace{x_{k}^{2}+y_{k}^{2}}_{d_{j k}^{2}} .
$$

Assim, das equações (2), (7) e (9), temos:

$$
d_{t k}^{2}=d_{i t}^{2}-2\left(x_{t} x_{k}+y_{t} y_{k}\right)+d_{i k}^{2} .
$$

Isolando o termo $x_{t}$ na equação (11): 


$$
x_{t}=\frac{d_{i t}^{2}+d_{i k}^{2}-d_{t k}^{2}-2 y_{t} y_{k}}{2 x_{k}} .
$$

Na equação (12), $x_{k}$ e $y_{k}$ foram determinados pelo resultado preliminar provado anteriormente, e $y_{t}$ é dado por (10), finalizando, portanto, a prova de que qualquer ponto $P_{t}$ é determinado univocamente pelas distâncias duas a duas entre as quatro cidades $i, j, k$ e $t$.

\section{Considerações Finais}

O resultado aqui demonstrado permite-nos, hipoteticamente, fazer um programa de computador a partir de três sensores que meçam a distância entre pontos para fazer o mapeamento de um ambiente. Como exemplo de aplicação, poderíamos citar a Agrimensura, que traz em si um conjunto de técnicas topográficas utilizadas para efetuar medidas de terras. Os sistemas de radares de trânsito ou outro qualquer tipo de rastreamento eletrônico constituem outros exemplos de aplicações para algoritmos semelhantes a esse.

\section{Referências}

[1] Barbosa, J.L.M. Geometria Plana Euclidiana. SBM,11ª Edição. Rio de Janeiro, 2012.

[2] Lima, E.L; Carvalho, P.C.P.; Wagner, E.;Morgado, A.C. A Matemática do Ensino Médio Volume 3. SBM, 6 ${ }^{\text {a }}$ Edição. Rio de Janeiro, 2006.

Josimar da Silva Rocha $\mathrm{UFTPR} / \mathrm{CP}$ $<$ jsrocha74@gmail.com>

Rogério César dos Santos Universidade de Brasília - UnB $<$ rogerc@unb.br>

Hudson Rodrigues Armando Universidade de Brasília - UnB $<$ hrodrigues1729@hotmail.com>

Recebido: 26/05/2019

Publicado: 16/08/2019 